
398.

\title{
Monitoring and Measuring Sedentary Behaviour with the Aid of Human Digital Memories
}

\author{
Chelsea Dobbins, Paul Fergus, Madjid Merabti, David Llewellyn-Jones \\ School of Computing and Mathematical Sciences \\ Liverpool John Moores University \\ Liverpool, UK \\ C.M.Dobbins@2006.ljmu.ac.uk, \{P.Fergus, M.Merabti, D.Llewellyn-Jones\}@ljmu.ac.uk
}

\begin{abstract}
There is growing global concern over the growing levels of obesity and the fact that people in general are not as active as they once were. Many believe that this is directly related to poor diet and our increasing reliance on technology, such as television, social networking, computer games, and voice activated home control systems. These kinds of activities increase sedentary behaviour across all age groups and is considered one of the main contributors to obesity and poor health. For this reason decreasing sedentary behaviour is considered a crucial theme within many research programs in health. Ironically, there is general agreement that the use of technology is likely to help researchers understand this type of behaviour. One interesting approach is based upon the use of human digital memories to provide visual lifelogs of a user's activity and to identify the behaviour patterns of individuals. In this way visual lifelogs provide a way for user's to evaluate their lifestyle choices. This paper discusses some of the key technologies used to achieve this and considers some of the challenges that still need to be addressed.
\end{abstract}

Index Terms-Lifelogging, Sedentary Behaviour, Digital Memory, Sensor

\section{INTRODUCTION}

Sedentary behaviour is broadly described as the amount of time we spend doing non-occupational activities, such as sitting, lying down and watching TV. This type of behaviour can be associated with excess weight gain and an increased risk of other diseases in later life, such as metabolic disorders and memory loss [1]. Whilst this behaviour does not seem particularly dangerous in our younger years the affect of little or no exercise, poor diet and alcohol misuse, becomes evident in later life, as the premature worsening of an individual's cognitive and physical capabilities are something that can occur over decades, as opposed to being evident over a number of days, weeks or months. This significantly impacts on our ability to live better during older age [2]. As the life expectancy of adults in Great Britain is increasing so is the occurrence of illness and disability [3]. As a result research into sedentary behaviour is growing rapidly, with early results indicating a potentially important negative health outcome for various markers of sedentary behaviour [4].

In order to reduce sedentary behaviour, so that the onset of these diseases can be prevented, awareness and changes towards our life style choices are essential. Therefore, for us to enjoy our later years, without debilitating illnesses, monitoring and measuring our behaviour is necessary. In this way it would give us an indication of our wellbeing and the impact lifestyle choices can have in later life.

The area of human digital memories has placed considerable focus on documenting the things we do, the places we visit and the thoughts we think [5]. This outlet allows us to capture, from a variety of data sources, rich information about ourselves and our surrounding environment and is an ideal platform to monitor our health and wellbeing. Monitoring sedentary behaviour, with the use of digital memories, not only provides a visual illustration of the state of our health and wellbeing at a particular moment in time, but could also act as a device to predict what our health would be like if we continued on the path we are on. We envision that the use of digital memories to influence changes in our sedentary behaviour is essential because if we could see and predict the state of our future health this evidence would serve as motivation in order for us to change our lifestyle. As stated by Ouellette and Wood [6], "It is through lack of uncertainty and immediate evidence that people fail to adopt a healthier lifestyle. To maintain intentions to adopt a healthier lifestyle, change strategies should ensure that some immediate, positive consequences emerge from the new healthy behaviour". We envision that these "positive consequences" could relate to our work within the field of digital memories [5]. By providing a visual depiction of how these changes could result in a healthier future, would be the incentive individuals need to change their lifestyles.

Given the significant impact digital technologies like smartphones, digital cameras and social networks, already have on our day-to-day living, it would be useful to consider their use in human digital memories research as a tool for monitoring and measuring sedentary behaviour. This paper explores the difficulties and possible solutions for achieving this.

\section{Capturing Human Digital Memories}

Capturing human digital memories, or the process of "lifelogging", is the practice of automatically recording one's life in digital form [7]. It presents an easy and unobtrusive way to document one's everyday life, without too much difficulty. 
C. Dobbins, P. Fergus, M. Merabti, and D. Llewellyn-Jones, "Monitoring and Measuring Sedentary Behaviour with the Aid of Human Digital Memories," in The 9th IEEE Consumer Communications \& Networking Conference (CCNC'12), 2012, pp. 395398.

One such initiative is a technology revolutionised by Microsoft called SenseCam, which was originally developed as a retrospective memory aid [8]. SenseCam has been used in a number of different applications, including applications to monitor sedentary behaviour. For example, Lindley et al. 's [9] study has focused on how technologies, such as SenseCam could be used to reflect upon the daily lives of people. The results from this study were positive and were supported by Doherty et al. [7] who state, "after participants looked at their sedentary images, they were prompted to change their lifestyle by, for example, cycling instead of driving, taking up exercise, and spending more time interacting with their children".

Many of the studies that have been undertaken have only monitored users for a short period of time [9-11]. Nonetheless, the study undertaken by Doherty et al. [7] has been particularly useful for developing automatic classifiers for visual life-logs to infer different lifestyle traits or characteristics. The study included 95 thousand manually annotated images and 3 million lifelog images obtained from 33 individuals sporadically over a period of 3.5 years. These images were later used to identify 22 different lifestyle traits [7] and to inform individuals about their future wellbeing using the analysis of these traits. Taking an opposing view, the study carried out by Kelly et al. [10] used the SenseCam to investigate its effectiveness within the area of travel research. Their initial results indicate that the overreporting of self-reported journeys was common and that in order to accurately track sedentary behaviour the SenseCam was a useful tool.

Whilst solutions for monitoring sedentary behaviour are likely to include digital memory technologies to address the problems with self-reporting, novel solutions are likely to include much richer data capturing capabilities to support the quantitative needs required in sedentary behaviour research as we shall see in the following section.

\section{Monitoring SEDEnTARy BeHAVIOUR}

The use of sensing technologies, to monitor and measure behaviour, is an area of interest that is being explored to help alleviate the limitations of self-reporting and to quantify different aspects of behaviour. One such initiative has been carried out by a research team using the activPAL single-axis accelerometer [11]. Sensors are placed on the thigh and the upper body to recognise sitting, lying or standing and walking transitions in healthy and community-dwelling older people without impairment. In a similar approach the activPAL accelerometer has been used to determine the habitual behaviour of people, whilst determining the interplay between sedentary behaviour and periods of physical activity [12]. However, the results from both of these studies indicate that it is difficult to accommodate slow gait, due to the small acceleration amplitudes generated, making its use with older age groups more difficult.

In a similar way pedometers have been used to measure physical activity $[14,15]$. In this instance pedometers have been used to monitor adherence in patients with cystic fibrosis, who

are generally encouraged to exercise regularly as part of their treatments. However, as reported in [13], pedometers have a number of limitations similar to accelerometers. Since they are primarily designed to detect vertical movement [14], i.e. walking, they are insensitive to sedentary behaviour, isometric activity, arm movements, including slow or fast walking velocities [15]. They also do not have any internal clock or data storage, which makes the analysis of activity patterns difficult to achieve [16].

Adopting a more ambitious approach the SenseWear Armband (SWA) houses several sensors within the device to collect data from bi-axial accelerometers, galvanic skin resistance sensors (sweat rates), heat flux sensors (heat dissipated from the body), and skin and near body temperature sensors [17]. The device has been used within Dwyer et al.'s [17] work to determine its accuracy in estimating energy expenditure (EE) and step count during treadmill walking in cystic fibrosis $(\mathrm{CF})$ patients, compared to healthy adults. Their initial results show that the SWA is capable of providing a reasonably accurate measure of step count compared to manual counting during treadmill walking [17]. In another study the SWA has been used to monitor adherence in women with rheumatoid arthritis to measuring decreases in sedentary behaviour [18]. The results from this study conclude that an $89 \%$ adherence rate was achieved and that the SWA was a viable method of quantifying physical activity [18].

Many variations of sensing and lifelogging technologies have been explored, however there still remains a number of significant problems that require further research as we shall see in the following section.

\section{OPEN RESEARCH CHALLENGES}

The literature shows that the use of technology to measure sedentary behaviour is mostly positive, yet it is reasonable to say that advanced solutions are needed. The following sections provide an overview of the key research challenges that need to be addressed in order to achieve this.

\section{A. Measurement}

Body Sensor Networking Platform: An extensible and open platform is required to support the independent use of sensors and through extended research, support for interconnecting and internetworking services that support much richer compositions (useful for determining context, i.e. there are several reasons why someone's heart rate has increased; running, being frightened, dread). It should provide a set of plug and play interfaces where for example, simple pressure sensors, and a combination of accelerometers and gyroscopes might be used. This data would then need to be interconnected with captured human digital memories in order to form a complete picture of an event.

Data Streams: Short term modelling of the data generated from real-time streams for reasoning may initially be quite straightforward to set up. However, in the longer term mathematical modelling and automated analysis of data 
C. Dobbins, P. Fergus, M. Merabti, and D. Llewellyn-Jones, "Monitoring and Measuring Sedentary Behaviour with the Aid of Human Digital Memories," in The 9th IEEE Consumer Communications \& Networking Conference (CCNC'12), 2012, pp. 395398.

streams through casual situations (stream reasoning [22-24]) may provide a more scalable strategy. Reasoning can be made over situations with transitions between situations given as successor state axioms as in [19]. In this way a sequence of casual actions is established that may govern behaviour and map sensed data to consequences.

Measuring Behaviour: The main challenge in measuring behaviour is to divide it into segments so that we can understand how they relate to each other. Furthermore, making distinctions between possibly similar behaviours under different contexts poses further challenges given that this affects segments and alters behaviours accordingly. It is variability within behaviour segments that makes classification difficult [20]. Clustering these similar events into activities, in order to build a lifelog, as in [21], also poses a challenge when incorporating sensor data to understand their relation to each other. However, since digital memories are comprised of so much more data than simply photos the clustering algorithms used would have to be extended beyond this scope and comprise of other forms of data as well, such as audio, video and physiological data so that our memories can be enhanced and these clusters can form a richer snapshot of our lives [5].

Classification of Behaviour: Using sensors provides streamed events from any number of data sources produced by the different body streams, allowing specific episodic manifestations to be monitored, e.g. sitting, walking or running. Mapping isolated data streams and their relationships with other data streams and then to higher order behaviours is nontrivial. The use of ontologies is one area that could potentially aid in this task. The Web Ontology Language (OWL) is an ontology language that provides classes, properties, class instances and data values [22] and provides a means of defining concepts from elements. It would be useful to explore whether this formulisation could be used to guide the mapping algorithms needed to link functions with behaviour where filtered data, provided by sensors can be used as inputs to ontological structures.

\section{B. Monitoring}

Time: Time is an important factor when monitoring patients as too little observation will render false results. As previously discussed many of the studies that have been undertaken have only monitored users for a short period of time [9-11]. For any health system to be effective prolonged monitoring is essential.

Processing: As a consequence of monitoring a user, essentially for their entire lives, will be the tremendous amounts of data generated. Automatically flagging significant events is a significant challenge. In terms of monitoring elderly patients, if a fall occurred or prolonged periods of sedentary behaviour was a common occurrence, these could be automatically flagged and shared between the patient and their carers to either determine the behaviour that led them to fall or to highlight a particular sedentary routine. At the other end of the spectrum, when monitoring younger adults or adolescents, lifelogs can aid in illustrating how their current lifestyle is impacting on their

future health. As identified above, data streams could be a way to order a sequence of casual actions to establish behaviour that may govern and map sensed data to consequences. Lifelogs can highlight a behaviour that might have been overlooked previously.

Structured Data: Another challenge is structuring the data in a way that aids the search and retrieval of content. One possibility, which we intend to address in our work, is semantically linking many data sources together to form richer memories that can be searched through more easily. We intend to create a system that will bring together and encompass various data sources so that a vivid interactive snapshot of our lives can be captured, reasoned upon and searched through. Interaction with our memories is fundamental to our ideas and is what makes it unique. By enabling user's to be able to "go into" their memories and to see various data, such as temperature, location and emotions, could lead to the augmentation of group memories and have the potential to benefit various aspects of people's lives [5].

Addressing the practicality of building a technological framework and its operation to support independent living in older adult's raises many questions, such as, is it feasible to monitor someone for their entire life? Whilst technology can point out our shortcomings, in terms of sedentary behaviour and the lifestyle choices we make, this begs the question whether it is enough of a motivation for individuals to change their lifestyle or whether human behaviour can ever be reasoned upon from sensor readings? These are important points to consider and one's which will be driving this research forward.

\section{CONCLUSION AND FUTURE WORK}

Sedentary behaviour is a growing problem amongst all age groups. Preventing the onset of certain diseases and generally improving our quality of life by monitoring and measuring sedentary behaviour, using the medium of digital memories, has been the focus of this paper. If we could provide a way to highlight to people the amount of time they spend in periods of inactivity this might provide the evidence they need to change their behaviour. We feel that our work would especially help elderly patients to prevent the onset of debilitating diseases, by providing information to their careers and the individuals themselves, about their behaviour patterns and to increase their activity levels. In observational studies, it has been noted that elderly persons who had participated to a greater extent in leisure activities had a lower risk of diseases, such as dementia, than those who had participated to a lesser extent [23]. Our future work aims to build on the ideas presented in this paper to help monitor and measure sedentary behaviour.

\section{REFERENCES}

[1] B. K. Clark, T. Sugiyama, G. N. Healy, J. Salmon, D. W. Dunstan, and N. Owen, "Validity and Reliability of Measures of Television Viewing Time and Other Non-Occupational Sedentary Behaviour of Adults: A Review," Obesity Reviews : An Official Journal of the International Association for the Study of Obesity, vol. 10, no. 1, pp. 7-16, Jan. 2009. 
C. Dobbins, P. Fergus, M. Merabti, and D. Llewellyn-Jones, "Monitoring and Measuring Sedentary Behaviour with the Aid of Human Digital Memories," in The 9th IEEE Consumer Communications \& Networking Conference (CCNC'12), 2012, pp. 395398.

[2] S. M. Strayer, J. R. Martindale, S. L. Pelletier, S. Rais, J. Powell, and J. B. Schorling, "Development and Evaluation of an Instrument For Assessing Brief Behavioral Change Interventions.," Patient education and counselling, vol. 83, no. 1, pp. 99-105, Apr. 2011.

[3] Office for National Statistics, "Healthy Life Expectancy: Living Longer In Poor Health," 2010. [Online]. Available: http://www.statistics.gov.uk/cci/nugget.asp?id=2159.

[Accessed: Aug-2011].

[4] S. J. H. Biddle, N. Pearson, G. M. Ross, and R. Braithwaite, "Tracking of Sedentary Behaviours of Young People: A Systematic Review," Preventive Medicine, vol. 51, no. 5, pp. 345-51, Nov. 2010.

[5] C. Dobbins, M. Merabti, P. Fergus, and D. Llewellyn-Jones, "Towards a Framework for Capturing and Distributing Rich Interactive Human Digital Memories," in 12th Annual Postgraduate Symposium on the Convergence of Telecommunications, Networking and Broadcasting (PGNet 2011), Liverpool, UK, 27-28 June 2011, 2011.

[6] J. A. Ouellette and W. Wood, "Habit and Intention In Everyday Life: The Multiple Processes By Which Past Behavior Predicts Future Behavior," Psychological Bulletin, vol. 124(1), pp. 54-74, 1998.

[7] A. R. Doherty et al., "Passively Recognising Human Activities Through Lifelogging," Computers in Human Behavior, vol. 27, no. 5, pp. 1948-1958, Sep. 2011.

[8] S. Hodges et al., "SenseCam: A Retrospective Memory Aid," UbiComp 2006: Ubiquitous Computing, vol. 4206, pp. $177-$ 193, 2006.

[9] S. E. Lindley, D. Randall, W. Sharrock, M. Glancy, N. Smyth, and R. Harper, "Narrative, Memory and Practice: Tensions and Choices in the Use of a Digital Artefact," Proceedings of the 23rd British HCI Group Annual Conference on People and Computers: Celebrating People and Technology, pp. 1-9, Sep. 2009.

[10] P. Kelly, A. Doherty, E. Berry, S. Hodges, A. M. Batterham, and C. Foster, "Can We Use Digital Life-Log Images To Investigate Active And Sedentary Travel Behaviour? Results From A Pilot Study," The International Journal of Behavioral Nutrition and Physical Activity, vol. 8, no. 1, p. 44, Jan. 2011.

[11] K. Taraldsen et al., "Evaluation of a Body-Worn Sensor System to Measure Physical Activity in Older People With Impaired Function," Physical Therapy: Journal of the American Physical Therapy Association, vol. 91, no. 2, pp. 277-285, Feb. 2011.

[12] S. Lord, S. F. M. Chastin, L. McInnes, L. Little, P. Briggs, and L. Rochester, "Exploring Patterns of Daily Physical and
Sedentary Behaviour in Community-Dwelling Older Adults," Age and Ageing, vol. 40, no. 2, pp. 205-210, 2011.

[13] B. S. Quon et al., "Monitoring Habitual Physical Activity In Cystic Fibrosis Patients Using Pedometers," American Journal of Respiratory and Critical Care Medicine, vol. 183, no. 1, p. A1121, May. 2011.

[14] F. Pitta, T. Troosters, V. S. Probst, M. A. Spruit, M. Decramer, and R. Gosselink, "Quantifying Physical Activity in Daily Life With Questionnaires and Motion Sensors in COPD," The European Respiratory Journal: Official Journal of the European Society for Clinical Respiratory Physiology, vol. 27, no. 5, pp. 1040-55, May. 2006.

[15] S.-I. Ao et al., "Review of Daily Physical Activity Monitoring System Based on Single Triaxial Accelerometer and Portable Data Measurement Unit," Machine Learning and Systems Engineering, vol. 68, pp. 569-580, 2010.

[16] D. R. Bassett, "Validity and Reliability Issues in Objective Monitoring of Physical Activity," Research Quarterly for Exercise and Sport, vol. 71, no. 2, p. S30-S36, 2000.

[17] T. J. Dwyer, J. A. Alison, Z. J. McKeough, M. R. Elkins, and P. T. P. Bye, "Evaluation of the SenseWear activity monitor during exercise in cystic fibrosis and in health.," Respiratory Medicine, vol. 103, no. 10, pp. 1511-1517, Oct. 2009.

[18] G. J. M. Almeida, M. C. M. Wasko, K. Jeong, C. G. Moore and S. R. Piva, "Physical Activity Measured by the SenseWear Armband in Women With Rheumatoid Arthritis.," Physical Therapy: Journal of the American Physical Therapy Association, Jun. 2011.

[19] R. Demolombe and M. del Pilar Pozos Parra, "A Simple and Tractable Extension of Situation Calculus to Epistemic Logic," pp. 515-524, Jul. 2000.

[20] J. C. Fentress, "Streams and patterns in behavior as challenges for future technologies.," Behavior research methods, vol. 41, no. 3, pp. 765-71, Aug. 2009.

[21] K. Petridis et al., "Knowledge Representation and Semantic Annotation of Multimedia Content," IEE Proceedings Vision, Image, and Signal Processing, vol. 153, no. 3, p. 255, 2006.

[22] W3C, "OWL 2 Web Ontology Language Document Overview," 2009. [Online]. Available: http://www.w3.org/TR/owl2-overview/. [Accessed: Aug2011].

[23] J. Verghese et al., "Leisure Activities and the Risk of Dementia in the Elderly," New England Journal of Medicine, pp. 2508-2516, 2003. 